\title{
El teléfono móvil inteligente: una herramienta para el estudio de la acústica experimental
}

Marcos H. Giménez Valentín ${ }^{\mathrm{a}, \mathrm{b}}$, Isabel Salinas Marín ${ }^{\mathrm{b}}$, Juan A. Monsoriu Serrab, V. P. Cuenca-Gotor ${ }^{b}$, F.J. Manjón ${ }^{b, c}$, J.A. Sans ${ }^{c}$ y J.A. Gómez-Tejedor ${ }^{b}$

a mhgimene@fis.upv.es, ${ }^{\mathrm{b}}$ Departamento de Física Aplicada, Escuela Técnica Superior de Ingeniería del Diseño, Universitat Politècnica de València, Camino de Vera s/n, 46022 Valencia, Spain, ${ }^{\mathrm{c}}$ Instituto de Diseño para la Fabricación y Producción Automatizada, Universitat Politècnica de València, Camino de Vera s/n, 46022 Valencia, Spain.

\begin{abstract}
In this work, we detail a new application of the use of the smartphone in the laboratory of Physics, in order to show the students that new technologies and the Physics' basic principles are compatible.

From the set of sensors that a mid-range mobile phone has, we have chosen the microphone for this study. We have designed an experience of sound waves superposition (acoustic beat) which combines the use of the common instrumentation of a Physics laboratory, such as current generators and speakers, and the smartphone as a measuring tool.

Using a simple free application designed for Android ${ }^{T M}$ smartphones, one can measure the acoustic intensity as a function of time and then export registered data to a csv file format. Applying common graphic and adjustment tools, the frequency of the beat generated by the superposition of two sinusoidal acoustic waves, with equal intensity and close frequencies, can be obtained in order to check that the difference between the two frequencies matches the acoustic beat frecuency.
\end{abstract}

Keywords: smartphone, Physics, acoustics, laboratory experiment.

\footnotetext{
Resumen

En este trabajo se detalla una nueva aplicación del uso del teléfono móvil "inteligente" (smartphone) dentro del laboratorio de Física, con objeto de mostrar a los alumnos que las nuevas tecnologías y los principios básicos de la Física no son universos excluyentes.
} 
El teléfono móvil inteligente: una herramienta para el estudio de la acústica experimental

De entre los diversos sensores que tiene un teléfono móvil de gama media, se ha trabajado con el micrófono. Se ha diseñado una práctica de superposición de ondas sonoras (batido acústico) en la que junto a elementos habituales de un laboratorio de Física, como son los generadores de corriente y los altavoces, se incluye el teléfono móvil como herramienta de medida.

A través de una sencilla aplicación gratuita desarrollada para teléfonos con sistema operativo Android ${ }^{T M}$, que mide la intensidad acústica en función del tiempo, se puede exportar los datos registrados a un fichero con formato csv. A partir de estos datos, y aplicando las herramientas de gráficas y ajustes habituales, se puede obtener la frecuencia del batido generado por la superposición de dos ondas acústicas sinusoidales de igual intensidad y de frecuencias próximas, y comprobar que la diferencia entre estas frecuencias coincide con la del batido acústico.

Palabras clave: smartphone, Física, acústica, práctica laboratorio.

\section{Introducción}

Los dispositivos móviles, a través de los sensores que llevan integrados, ofrecen nuevas oportunidades para la realización de sencillos experimentos de Física para los primeros cursos de Grado. Por ejemplo, diversos procesos físicos pueden ser analizados mediante el uso de una cámara digital utilizando los vídeos grabados para medir los intervalos de tiempo, distancias y trayectorias de objetos en movimiento (Monsoriu, 2005). También dispositivos inalámbricos, como el wiimote, se utilizan en la enseñanza de la Física (Tomarken, 2012). Estos aparatos tienen un acelerómetro de tres ejes que se comunica con la consola de juegos por medio de un dispositivo Bluetooth ${ }^{\mathrm{TM}}$. Más recientemente, los smartphones o teléfonos inteligentes se han incorporado en esta variedad de dispositivos portátiles (Sans, 2013). Así, por ejemplo, el sensor de aceleración de los smartphones se ha utilizado para el estudio de las oscilaciones, tanto a nivel cualitativo (Kuhn, 2012) como cuantitativo (Castro-Palacio, 2013). Estos trabajos muestran experimentos muy sencillos donde el teléfono móvil es el objeto en movimiento y cuya aceleración es registrada a través del sensor de aceleración que lleva integrado el propio teléfono.

En el presente trabajo se expone una nueva experiencia con los smartphones para el estudio experimental del batido acústico. Este fenómeno tiene lugar cuando se superponen dos ondas acústicas de frecuencia próxima y, entre otras aplicaciones, permite afinar instru- 
Marcos H. Giménez Valentín, Isabel Salinas Marín, Juan A. Monsoriu Serra, V.P. Cuenca-Gotor, F.J. Manjón, J.A. Sans y J.A. Gómez-Tejedor

mentos de música (Tipler, 2005). En este trabajo se han generado dos ondas sonoras armónicas de frecuencia próxima con dos altavoces, cada uno de los cuales estaba conectado a un generador de tensión alterna distinto. Gracias a una aplicación gratuita desarrollada para teléfonos con sistema operativo Android ${ }^{\mathrm{TM}}$, es posible utilizar el smartphone como sonómetro de precisión. De esta forma, los alumnos pueden registrar de forma sencilla el batido acústico resultante y determinar su frecuencia de pulsación. Los resultados obtenidos a partir del smartphone (frecuencia de batido o de pulsación) concuerdan con las predicciones teóricas (resta de las frecuencias de los sonidos superpuestos).

\section{Objetivos}

- Enseñar al alumno que se puede utilizar el teléfono móvil como un instrumento de medida haciendo uso de aplicaciones gratuitas, como "Physics Toolbox Sound Meter" desarrollada para Android ${ }^{\mathrm{TM}}$, que es capaz de medir el nivel sonoro (en decibelios) en función del tiempo, mostrando gráficamente en la pantalla su evolución, y de exportar esas medidas a un ordenador; e.g., mediante correo electrónico o a través de una conexión Bluetooth ${ }^{\mathrm{TM}}$.

- Integrar en el laboratorio de Física un elemento de uso cotidiano, como es el teléfono móvil "inteligente" (smartphone) de gama media, del que dispone un alto porcentaje de alumnos.

- Analizar el fenómeno del batido acústico que se produce al superponer dos sonidos armónicos de la misma amplitud, pero con frecuencias ligeramente distintas.

\section{Desarrollo de la innovación}

\subsection{Fundamento teórico}

Sean dos oscilaciones armónicas simples, $x_{1}$ y $x_{2}$, de igual amplitud $A$, frecuencias próximas $f$ y $f+\Delta f$, y fases iniciales $\varphi_{1}$ y $\varphi_{2}$, de modo que:

$$
\begin{aligned}
& x_{1}=A \operatorname{sen}\left[2 \pi f t+\varphi_{1}\right] \\
& x_{2}=A \operatorname{sen}\left[2 \pi(f+\Delta f) t+\varphi_{2}\right]
\end{aligned}
$$

Si estas dos oscilaciones se superponen, se tiene que:

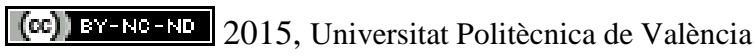

Congreso In-Red (2015) 


$$
\begin{aligned}
& x=x_{1}+x_{2}= \\
& =A \operatorname{sen}\left[2 \pi f t+\varphi_{1}\right]+A \operatorname{sen}\left[2 \pi(f+\Delta f) t+\varphi_{2}\right]= \\
& =A 2 \operatorname{sen} \frac{\left[2 \pi(f+\Delta f) t+\varphi_{2}\right]+\left[2 \pi f t+\varphi_{1}\right]}{2} \cos \frac{\left[2 \pi(f+\Delta f) t+\varphi_{2}\right]-\left[2 \pi f t+\varphi_{1}\right]}{2}= \\
& =2 A \operatorname{sen} \frac{2 \pi(2 f+\Delta f) t+\varphi_{2}+\varphi_{1}}{2} \cos \frac{2 \pi \Delta f t+\varphi_{2}-\varphi_{1}}{2}= \\
& =2 A \operatorname{sen}\left[2 \pi\left(f+\frac{\Delta f}{2}\right) t+\frac{\varphi_{2}+\varphi_{1}}{2}\right] \cos \frac{2 \pi \Delta f t+\varphi_{2}-\varphi_{1}}{2}= \\
& =A^{\prime} \operatorname{sen}\left[2 \pi\left(f+\frac{\Delta f}{2}\right) t+\frac{\varphi_{2}+\varphi_{1}}{2}\right]
\end{aligned}
$$

El resultado equivale a una oscilación cuya frecuencia es la media de las de las oscilaciones superpuestas, y con una amplitud $A^{\prime}$ que varía con el tiempo de acuerdo con la expresión:

$$
A^{\prime}=2 A \cos \frac{2 \pi \Delta f t+\varphi_{2}-\varphi_{1}}{2}
$$

Si las oscilaciones $x_{1}$ y $x_{2}$ se deben a sendas ondas que interfieren en un cierto punto del espacio, la intensidad $I$ de esa interferencia en dicho punto es proporcional al cuadrado de la amplitud resultante. Denominando $\lambda$ al factor de proporcionalidad, resulta:

$$
\begin{aligned}
I & =\lambda A^{\prime 2}=\lambda 4 A^{2} \cos ^{2} \frac{2 \pi \Delta f t+\varphi_{2}-\varphi_{1}}{2}= \\
& =2 \lambda A^{2}\left(1+\cos \left[2 \pi \Delta f t+\varphi_{2}-\varphi_{1}\right]\right)= \\
& =2 \lambda A^{2}+2 \lambda A^{2} \cos \left[2 \pi \Delta f t+\varphi_{2}-\varphi_{1}\right]
\end{aligned}
$$

Por tanto la intensidad oscila, alrededor de un valor medio, con una frecuencia que es la diferencia de las de las ondas que interfieren. La figura 1 muestra un ejemplo en el que $x_{1}$ y $x_{2}$ son las oscilaciones debidas a dos ondas de desplazamientos de la misma amplitud, $A=1 \mathrm{~m}$, frecuencias próximas $f=10 \mathrm{~Hz}$ y $f+\Delta f=10+1=11 \mathrm{~Hz}$, y fases iniciales $\varphi_{1}=\varphi_{2}=0$ rad. La frecuencia del cuadrado de la amplitud $A^{\prime}$ (y por tanto la de la intensidad $I=\lambda A^{\prime 2}$ ) es $\Delta f=1 \mathrm{~Hz}$.

Cuando las ondas que interfieren son acústicas, como es el caso del sonido captado por un micrófono, todo lo anterior es aplicable si ambas ondas llegan a él con igual amplitud. Con precisión suficiente, ésta es la situación que se tiene cuando el micrófono está situado a igual distancia de dos altavoces idénticos, alimentados por corrientes alternas sinusoidales producidas por diferencias de tensión alternas de frecuencias próximas e igual tensión eficaz. 
Marcos H. Giménez Valentín, Isabel Salinas Marín, Juan A. Monsoriu Serra, V.P. Cuenca-Gotor, F.J. Manjón, J.A. Sans y J.A. Gómez-Tejedor
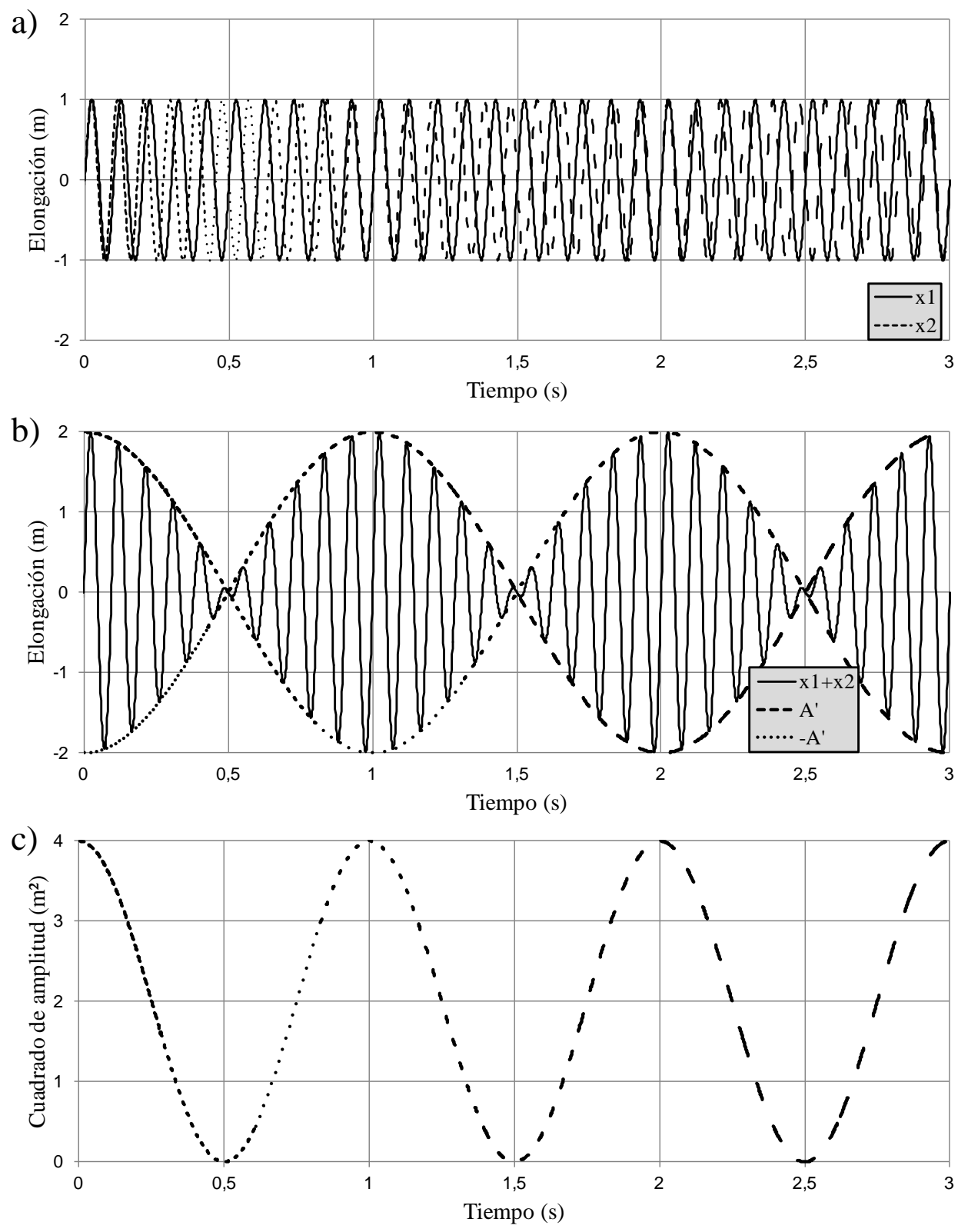

Fig. 1. Superposición de dos oscilaciones $x_{1}$ y $x_{2}$ de la misma amplitud $(A=1 m)$ y de frecuencias próximas $(f=10 \mathrm{~Hz} ; f+\Delta f=10+1=11 \mathrm{~Hz})$ : a) oscilaciones individuales; $b)$ superposición de las oscilaciones y amplitud envolvente; c) cuadrado de la amplitud. 
El teléfono móvil inteligente: una herramienta para el estudio de la acústica experimental

\subsection{Montaje experimental}

El sistema para la realización del experimento, mostrado en la figura 2, consta de dos generadores de corriente alterna (en nuestro caso, del modelo 33120A de Hewlett-Packard), dos altavoces genéricos idénticos enfrentados, y los pertinentes cables para conectar cada altavoz a su generador de corriente. Finalmente, el teléfono móvil se coloca en la posición intermedia entre los altavoces. Previamente, se ha instalado en el teléfono móvil la aplicación capaz de medir el nivel sonoro en decibelios (en nuestro caso, "Physics Toolbox Sound Meter”, desarrollada para Android ${ }^{\mathrm{TM}}$ ) mediante su micrófono.

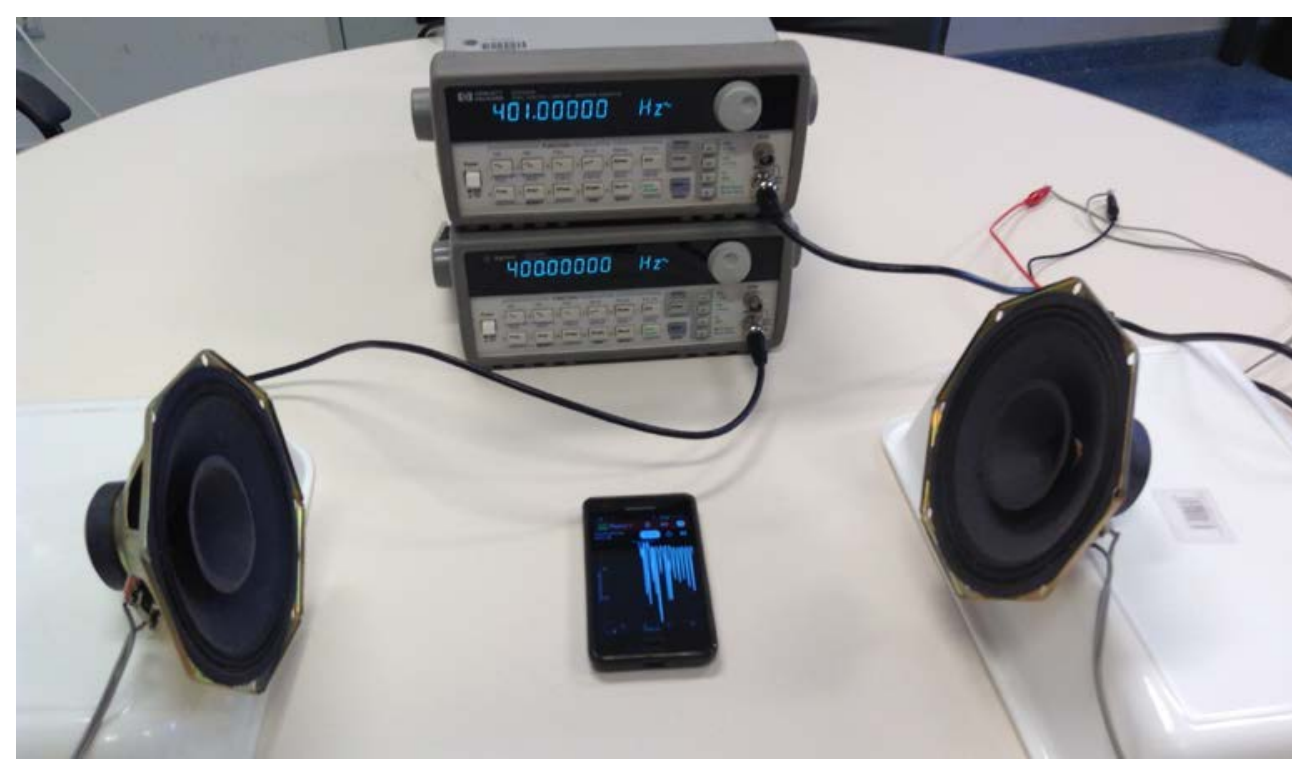

Fig. 2. Montaje experimental

\subsection{Realización del experimento}

En primer lugar se especifica una tensión común en los dos generadores (en nuestro caso, $V_{p p}=5 \mathrm{~V}$ ), y se configuran para que los dos altavoces conectados sean alimentados por corrientes alternas sinusoidales de frecuencias próximas y dentro del rango humano de audición (400Hz y $401 \mathrm{~Hz}$ en el ejemplo que se muestra en la figura 2). Tras comprobar que se oye un sonido de intensidad oscilante, el batido acústico, se activa en el teléfono móvil la aplicación para registrar el nivel sonoro (en decibelios), observándose sus fluctuaciones en la pantalla (véase la figura 3). Se puede verificar así que, aún cuando exista un pequeño nivel de ruido de fondo, y que la frecuencia de muestreo pueda no ser suficiente para capturar con precisión los mínimos de la señal, sí que se observa adecuadamente el ritmo periódico de las fluctuaciones. 
Marcos H. Giménez Valentín, Isabel Salinas Marín, Juan A. Monsoriu Serra, V.P. Cuenca-Gotor, F.J. Manjón, J.A. Sans y J.A. Gómez-Tejedor

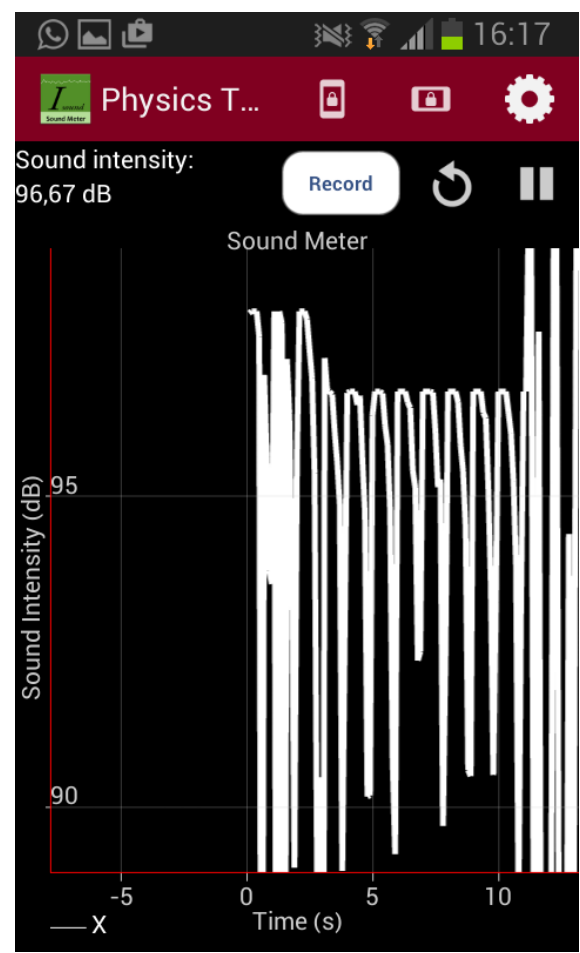

Fig. 3. Fluctuaciones del nivel sonoro del batido acústico, mostradas en la pantalla de un teléfono móvil que ejecuta la aplicación "Physics Toolbox Sound Meter".

Tras almacenar datos del nivel sonoro durante 30s, se pueden exportar en formato de fichero .csv. Este fichero se puede envíar al ordenador del laboratorio mediante la aplicación de correo electrónico del teléfono móvil, para posteriormente proceder a su análisis mediante una hoja de cálculo.

Para realizar el análisis, lo primero que hay que hacer es convertir la columna de datos del nivel sonoro $\beta$ (en $\mathrm{dB}$ ) a intensidad $I\left(\mathrm{en} \mathrm{W} / \mathrm{m}^{2}\right)$ mediante la expresión:

$$
I=I_{0} 10^{\beta / 10}
$$

donde $I_{0}=10^{-12} \mathrm{~W} / \mathrm{m}^{2}$ es el valor estándar de la intensidad umbral de la audición humana.

Posteriormente, se debe seleccionar un intervalo de unos 4 ó 5s de la parte central de toda la serie temporal de la intensidad. A continuación, se realiza un ajuste por mínimos cuadrados de esa serie de datos $I(t)$ a una función del tipo $y(t)=A_{0}+A \operatorname{sen}\left(\omega t^{+} \varphi\right)$, como la expresión (4). El ajuste proporciona los valores de los cuatro parámetros $\left(A_{0}, A, \omega \mathrm{y} \varphi\right)$, aunque el único relevante para el objetivo final del experimento es la frecuencia de la pulsación $\omega$. No obstante, la obtención de los cuatro parámetros permite representar la función $y(t)$ junto a la serie de datos $I(t)$ y verificar así visualmente la idoneidad del ajuste realizado. 
Hay que hacer constar que la función utilizada en el ajuste puede incluir la función seno, como en el párrafo anterior, o la función coseno, como se ha indicado en el apartado dedicado al fundamento teórico. En cualquier caso, utilizar una u otra función afecta únicamente al valor de la fase inicial $\varphi$, y por tanto no tiene relevancia en la obtención de la frecuencia del batido acústico.

A partir del valor del parámetro $\omega$ proporcionado por el ajuste, se puede determinar la frecuencia $f_{b}$ del batido acústico mediante la expresión:

$$
f_{b}=\frac{2 \pi}{\omega}
$$

Finalmente, se puede comparar la frecuencia $\mathrm{f}_{\mathrm{b}}$ obtenida del ajuste de datos experimentales con el valor $\Delta f$ en que difieren las frecuencias de los dos generadores de corriente.

a)
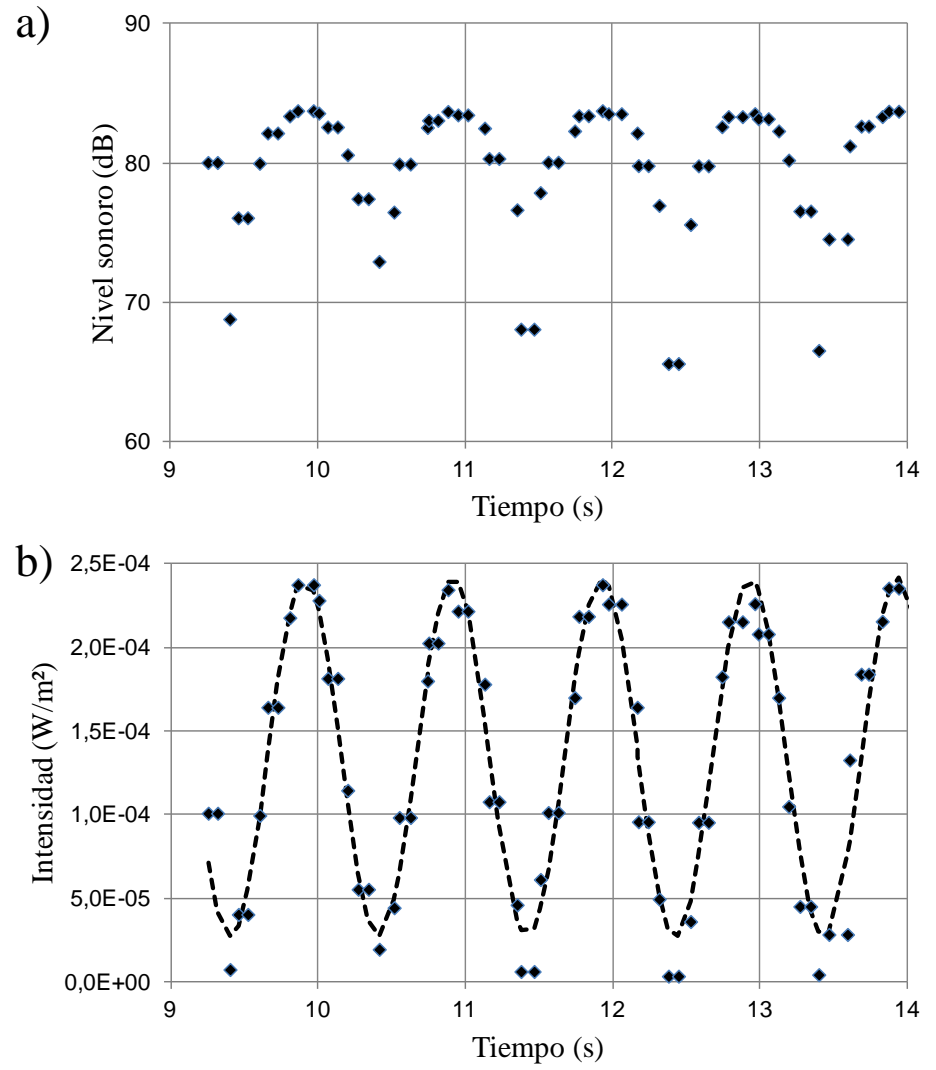

Fig. 4. Análisis a partir de los datos de sonido registrados por el teléfono móvil: a) serie temporal de nivel sonoro; b) serie temporal de intensidad del sonido (marcas) y forma funcional ajustada (línea a trazos). 
Marcos H. Giménez Valentín, Isabel Salinas Marín, Juan A. Monsoriu Serra, V.P. Cuenca-Gotor, F.J. Manjón, J.A. Sans y J.A. Gómez-Tejedor

Hay que mencionar que es conveniente repetir el experimento configurando los generadores de corriente para que proporcionen diversas combinaciones de corrientes alternas sinusoidales, siempre de frecuencias próximas, con objeto de comprobar que en todas ellas se verifica la concordancia $f_{b} \approx \Delta f$. Esto debe ocurrir siempre que $\Delta f$ sea lo suficientemente pequeño con respecto a las frecuencias de las corrientes.

La figura 4 muestra, como ejemplo, el resultado obtenido cuando las frecuencias de los sonidos son $f=700 \mathrm{~Hz}$ y $f+\Delta f=700+1=701 \mathrm{~Hz}$. En primer lugar, se representa un intervalo central de la serie temporal del nivel sonoro registrada por el teléfono móvil. A continuación, aparece la correspondiente serie temporal de intensidad del sonido y la función ajustada por el método de los mínimos cuadrados. Puede observarse que la calidad del ajuste es suficientemente buena como para probar que el proceso es periódico, y determinar por tanto su frecuencia.

\section{Resultados}

Como ejemplo, se han realizado ensayos con cuatro combinaciones de frecuencias de las corrientes alternas suministradas por los generadores de corriente, y por lo tanto de los sonidos que las mismas producen en los altavoces. El análisis de las series temporales registradas por el teléfono móvil, realizado mediante el procedimiento descrito anteriormente, proporciona las frecuencias de batido, $f_{b}$, que se muestran en la tabla 1 . Se pone así de manifiesto la gran concordancia con los valores teóricos $\Delta f$, ya que puede observarse que la discrepancia no supera el nivel del $1 \%$.

Tabla 1. Combinaciones de frecuencias utilizadas, y resultados

\begin{tabular}{cccc}
\hline Frecuencia generador 1 & Frecuencia generador 2 & $\boldsymbol{\Delta f \text { (teórica) }}$ & $\boldsymbol{f}_{\boldsymbol{b}}$ (experimental) \\
\hline $400 \mathrm{~Hz}$ & $401 \mathrm{~Hz}$ & $1 \mathrm{~Hz}$ & $0,994 \mathrm{~Hz}$ \\
$500 \mathrm{~Hz}$ & $501 \mathrm{~Hz}$ & $1 \mathrm{~Hz}$ & $1,010 \mathrm{~Hz}$ \\
$600 \mathrm{~Hz}$ & $601 \mathrm{~Hz}$ & $1 \mathrm{~Hz}$ & $0,994 \mathrm{~Hz}$ \\
$700 \mathrm{~Hz}$ & $701 \mathrm{~Hz}$ & $1 \mathrm{~Hz}$ & $0,992 \mathrm{~Hz}$ \\
\hline
\end{tabular}

En todo caso, aún cuando el grado de precisión de la determinación de la frecuencia de batido con el método descrito es razonable; cabe recordar que, además de analizar el fenómeno del batido acústico y de verificar el acuerdo entre la predicción teórica y el resultado experimental, uno de los principales objetivos del presente experimento es la integración

(c)) EY-NC-ND 2015, Universitat Politècnica de València

Congreso In-Red (2015) 
del teléfono móvil en el laboratorio con objeto de despertar la curiosidad del estudiante y aumentar su motivación.

\section{Conclusiones}

En este trabajo se ha presentado una nueva experiencia de laboratorio, enfocada a alumnos de primeros cursos universitarios, en la que se utiliza el sonómetro de un teléfono móvil "inteligente" (smartphone) para registrar la serie temporal de nivel sonoro de un batido acústico. Dicho batido se ha obtenido por medio de dos altavoces conectados a sendos generadores de tensión que producen corrientes alternas sinusoidales de igual amplitud, y frecuencias próximas.

El análisis de los datos de la serie temporal registrada permite a los alumnos obtener la frecuencia del batido acústico y comprobar que se corresponde casi exactamente (con una discrepancia que no supera el nivel del $1 \%$ en nuestras pruebas) con el valor predicho por la teoría. Además, el procedimiento utilizado supone para el alumno un ejemplo de cómo se puede utilizar el teléfono móvil en experiencias de Física, lo que persigue despertar su sorpresa y curiosidad y aumentar su grado de motivación.

\section{Referencias}

CASTRO-PALACIO, J.C., Velazquez-Abad, L., Giménez, M.H. y Monsoriu, J.A. (2013). “Using the mobile phone acceleration sensor in Physics experiments: free and damped harmonic oscillations" en American Journal of Physics, vol. 81, p. 472-475.

KUHN,J. y VOGT, P. (2012). “Analyzing spring pendulum phenomena with a smartphone acceleration sensor”, en The Physics Teacher, vol. 50, p. 504-505.

MONSORIU, J.A., GIMÉNEZ, M.H., RIERA, J. y VIDAURRE, A. (2005). "Measuring coupled oscillations using an automated video analysis technique based on image recognition”, en European Journal of Physics, vol. 26, p. 1149-1155.

SANS, J.A., MANJÓN, F.J., PEREIRA, J.A., GÓMEZ-TEJEDOR, J.A. y MONSORIU, J.A. (2013). "Oscillations studied with the smartphone ambient light sensor", en European Journal of Physics, vol. 34, p. 1349-1354.

TIPLER, P.A. y MOSCA, G. (2005). Física para la ciencia y la tecnología. Barcelona: Editorial Reverté.

TOMARKEN, S.L., SIMONS, D.R., HELMS, R.W., JOHNS, W.E., SCHRIVER, K.E. y WEBSTER, M.S. (2012). "Motion tracking in undergraduate physics laboratories with the Wii remote”, en American Journal of Physics, vol. 80, p. 351-354. 
Marcos H. Giménez Valentín, Isabel Salinas Marín, Juan A. Monsoriu Serra, V.P. Cuenca-Gotor, F.J. Manjón, J.A. Sans y J.A. Gómez-Tejedor

\section{Agradecimientos}

Los autores desean agradecer al Instituto de Ciencias de la Educación de la Universitat Politécnica de Valencia su apoyo en los grupos de innovación docente MoMA y e-MACAFI y por subvencionar el proyecto PIME/2014/A/031/B que ha dado lugar a este trabajo. 